\title{
Model comparisons and genetic parameter estimates of growth and the Kleiber ratio in Shal sheep
}

\author{
Hossein Mohammadi', Mohammad Moradi Shahrebabak², Hossein Moradi \\ Shahrebabak², Abolfazl Bahrami² and Mohammad Dorostkar ${ }^{1}$ \\ 'Department of Animal Science, College of Agriculture, University of Tabriz, Tabriz, Iran, ${ }^{2}$ Department of Animal \\ Science, College of Agriculture and Natural Resources, University of Tehran, Karaj, Iran
}

\section{Abstract}

Genetic and phenotypic parameters for lamb growth traits were estimated for the Shal sheep used as animal model. Data on lamb growth performance were extracted from available performance records at the Shal sheep Station in Qazvin, Iran. Studied traits were the body weight of lambs at birth, at three months of age as weaning weight, the six months weight, nine months weight, yearling weight, average daily gain from birth to weaning and Kleiber ratio from birth to weaning. Significant random effects for each trait were determined by fitting additive direct genetic effects, additive maternal effects, the covariance between additive direct and additive maternal effects, maternal permanent environmental and maternal temporary environmental (common litter) effects of twelve animal models. Univariate analyses were carried out under the most appropriate model, determined by the Akaike information criterion test. Direct heritability estimates for birth weight, weaning weight, average daily gain, Kleiber ratio, six months weight, nine months weight and yearling weight were $0.13,0.19,0.18,0.05,0.16,0.18$ and 0.19 , respectively. Maternal additive genetic effects were fitted only for birth weight and weaning weight. Corresponding estimates of 0.12 and 0.10 were obtained for maternal heritability of birth weight and weaning weight, respectively. Maternal permanent environmental effects have low contribution to the expression of Kleiber ratio and lead to estimates of 0.06 and 0.06 for permanent maternal environmental variance as a proportion of phenotypic variance $\left(c^{2}\right)$ of these traits, respectively. All pre-weaning traits, except Kleiber ratio, were affected by litter effects. The magnitude of ratio of common litter variance to phenotypic variance $\left(l^{2}\right)$ was $0.05,0.12$ and 0.14 for birth weight, weaning weight and average daily gain, respectively. Direct genetic 
correlations were positive and ranged from 0.09 for Kleiber ratio-yearling weight to 0.80 for weaning weight-average daily gain. Phenotypic correlations ranged from 0.18 for Kleiber ratio-yearling weight to 0.87 for weaning weight-average daily gain.

Keywords: average daily gain, common environmental effect, Kleiber ratio, genetic parameters

Abbreviations: BW: birth weight, WW: weaning weight, 6MW: six months weight, 9MW: nine months weight, YW: yearling weight, ADG: average daily gain, KR: Kleiber ratio, AIC: Akaike information criterion

\section{Introduction}

Mutton production in Iran, as the main source of red meat, does not satisfy the increasing demand of the consumers. Van Wyk et al. (2003) pointed out that sheep production systems have to be dynamic to satisfy the changing consumers' demands. So, enhancing the growth potential of lambs and production of more lambs per ewe are possible alternatives to increase meat production in any sheep breeding system (Miraei-Ashtiani et al. 2007). The Iranian sheep population comprises of about 27 breeds and ecotypes (Vatankhah et al. 2004). This genetic variation provides appropriate opportunities for improving breeding efficiency through breeding strategies such as crossbreeding that exploits breed diversities and breed complementary (Freking \& Leymaster 2004).

Shal breed, numbering about 1.5 million animals, is one of the most important meat breeds among Iranian sheep. They are well known for their large size, tolerance to climatic changes and capability to produce twining lambs. The breed is fat-tailed, its coat colour is predominantly sugary greyish and a small number of them is also black and white. This breed has a white spot on the forehead and both sexes are not polled. The origin of the Shal sheep is the Qazvin province and it is kept mainly to produce meat (Tavakolian 1999).

Growth rate and body weight of lambs at different ages have deterministic effects on the profitability of sheep production enterprises. Therefore, these traits may be taken into account as efficient selection criterions in any sheep breeding system (Tosh \& Kemp 1994). Such appropriate selective procedure requires accurate (co)variance components and genetic parameter estimates. Genetic parameters for growth traits of different sheep breeds have been reported (Prince et al. 2010, Di et al. 2011, Abegaz et al. 2010). The results of these studies have shown that the inclusion of maternal effects on the models considered for genetic analysis of growth traits, especially for pre-weaning traits, is of crucial importance. They confirmed that ignoring maternal effects leads to upward biased estimates for (co) variance components. Thus, accurate estimation of (co)variance components is a prerequisite for designing any breeding programme and genetic evaluation system. Because of paucity of such estimates for growth traits of Shal sheep in Iran, the main objective of the current research was the estimation of (co)variance components and corresponding genetic parameters for growth traits of Shal sheep with an emphasis on partitioning maternal effects. 


\section{Materials and methods}

\section{Animals and management}

The flock was managed semi-intensively. At birth time and/or within $24 \mathrm{~h}$ afterwards, lambs were weighed and ear-tagged. Lambs were kept indoors from mid-February to late April and manually fed. Afterwards, lambs were grazed on pastures of low quality and productivity. Supplemental feeding comprising dried alfalfa and barley grain was offered to the animals, particularly prior to mating and late pregnancy. Weaning was at approximately 120 days of age. The lambs were weaned at the same day but not necessarily at the same age. The female lambs were exposed to the rams at approximately 18 months of age. A controlled mating strategy was designed during mating period (early November to mid-December) and ewes were mated to fertile rams at the rate of 20 ewes per ram. Ewes were kept for a maximum of seven parities (until eight years of age) and rams for a maximum of two mating seasons. Breeding rams and ewes were selected mainly based on phenotypic characteristics such as visual body conformation at yearling age and nearest three generations of pedigree information on birth type of the lambs.

\section{Studied traits}

Data used in the present study were collected during an 11-year period from 1998 to 2009 at Agricultural Jahad Organization, Qazvin province of Iran. Investigated traits were body weights of lambs at birth (BW), three months of age as weaning weight (WW), six months of age (6MW), nine months of age (9MW), yearling age (YW), average daily gain from birth to weaning (ADG) and Kleiber ratio from birth to weaning (KR) defined as ADG/WW ${ }^{0.75}$ The structure of the data set used in the present study is presented in Table 1.

Table 1

Characteristics of the data structure

\begin{tabular}{lcccccccc}
\hline Traits & $\begin{array}{c}\text { No. of } \\
\text { Records }\end{array}$ & Mean & $\begin{array}{c}\text { Standard } \\
\text { deviation }\end{array}$ & $\begin{array}{c}\text { \% Coefficient } \\
\text { of variation }\end{array}$ & $\begin{array}{c}\text { No. of } \\
\text { dams }\end{array}$ & $\begin{array}{c}\text { No. of } \\
\text { sires }\end{array}$ & $\begin{array}{c}\text { No. of dams } \\
\text { with records }\end{array}$ & $\begin{array}{c}\text { No. of sires } \\
\text { with records }\end{array}$ \\
\hline BW, kg & 6221 & 3.46 & 0.83 & 19.87 & 4060 & 160 & 3476 & 134 \\
WW, kg & 4261 & 22.65 & 3.12 & 15.78 & 3497 & 145 & 2974 & 109 \\
ADG, g/day & 4261 & 213.21 & 48.93 & 22.53 & 3497 & 145 & 2974 & 109 \\
KR & 4261 & 20.36 & 2.47 & 13.05 & 3497 & 145 & 2974 & 109 \\
6MW, kg & 3112 & 34.14 & 5.29 & 16.80 & 2481 & 137 & 2186 & 101 \\
9MW, kg & 2370 & 46.36 & 9.92 & 15.59 & 1883 & 107 & 1762 & 99 \\
YW, kg & 2240 & 55.46 & 6.97 & 17.54 & 1325 & 102 & 1190 & 93 \\
\hline
\end{tabular}

\section{Statistical analysis}

\section{Fixed effects}

Significance testing of fixed effects to be included in the operational model for each trait, was carried out using the general linear model (GLM) procedure of the SAS v9 software package (SAS Institute Inc., Cary, NC, USA) and least squares means for each trait were 
obtained. Considered fixed effects in the analytical model were sex of lamb in 2 classes (male and female), birth year in 11 classes (1998-2009), dam age at lambing in 6 classes (2-7 years old), birth type in 3 classes (single, twin and triplet) and age of lamb at 3, 6, 9 and 12 months (in days) as a linear covariate for $\mathrm{WW}, 6 \mathrm{MW}, 9 \mathrm{MW}$ and $\mathrm{YW}$, respectively. The interactions between fixed effects were not significant and therefore excluded.

Estimation of (co)variance components and genetic parameters

(Co)variance components and corresponding genetic parameters for the studied traits were estimated using restricted maximum likelihood (REML) using ASReml (Gilmour et al. 2000). Tested models (in matrix notation) are as shown below:

$$
\begin{array}{lll}
y=X b+Z_{1} a+e & & \text { Model (1) } \\
y=X b+Z_{1} a+Z_{3} c+e & & \text { Model (2) } \\
y=X b+Z_{1} a+Z_{4} I+e & & \text { Model (3) } \\
y=X b+Z_{1} a+Z_{3} c+Z_{4} I+e & & \text { Model (4) } \\
y=X b+Z_{1} a+Z_{2} m+e & \operatorname{Cov}(a, m)=0 & \text { Model (5) } \\
y=X b+Z_{1} a+Z_{2} m+e & \operatorname{Cov}(a, m)=A \sigma_{a m} & \text { Model (6) } \\
y=X b+Z_{1} a+Z_{2} m+Z_{3} c+e & \operatorname{Cov}(a, m)=0 & \text { Model (7) } \\
y=X b+Z_{1} a+Z_{2} m+Z_{3} c+e & \operatorname{Cov}(a, m)=A \sigma_{a m} & \text { Model (8) } \\
y=X b+Z_{1} a+Z_{2} m+Z_{4} I+e & \operatorname{Cov}(a, m)=0 & \text { Model (9) } \\
y=X b+Z_{1} a+Z_{2} m+Z_{4} I+e & \operatorname{Cov}(a, m)=A \sigma_{a m} & \text { Model (10) } \\
y=X b+Z_{1} a+Z_{2} m+Z_{3} c+Z_{4} I+e & \operatorname{Cov}(a, m)=0 & \text { Model (11) } \\
y=X b+Z_{1} a+Z_{2} m+Z_{3} c+Z_{4} I+e & \operatorname{Cov}(a, m)=A \sigma_{a m} & \text { Model (12) }
\end{array}
$$

Where $y$ is a vector of records for traits studied; $b, a, m, c, l$ and $e$ are vectors of fixed, direct genetic, maternal genetic, maternal permanent environmental, maternal temporary environmental (common litter) effects and the residual effects, respectively. $X, Z_{1^{\prime}} Z_{2^{\prime}} Z_{3}$ and $Z_{4}$ are corresponding design matrices associating the fixed, direct genetic, maternal genetic, maternal permanent environmental and maternal temporary environmental effects to vector of $y$.

It is assumed that direct additive genetic, maternal additive genetic, maternal permanent environmental, maternal temporary environmental and residual effects are normally 
distributed with mean 0 and variance $A \sigma^{2}{ }_{a^{\prime}} A \sigma^{2}{ }_{m^{\prime}} I_{d} \sigma_{c^{\prime}}^{2}, \sigma^{2}$ and $I_{n} \sigma_{e^{\prime}}^{2}$ respectively. Furthermore, $\sigma_{a^{\prime}}^{2} \sigma_{m^{\prime}}^{2} \sigma_{c^{\prime}}^{2} \sigma_{l}^{2}$ and $\sigma_{e}^{2}$ are direct additive genetic variance, maternal additive genetic variance, maternal permanent environmental variance (half sibs across years), maternal temporary environmental variance (full sibs within year) and residual variance, respectively. $A$ is the additive numerator relationship matrix; $I_{d^{\prime}} l_{l}$ and $I_{n}$ are identity matrices that have order equal to the number of dams, litters and number of records, respectively and $\sigma_{a m}$ refers to the covariance between direct genetic and maternal genetic effects.

An Akaike information criterion (AIC) test was applied to determine the most appropriate model for estimating (co)variance components for each traits as follows (Akaike 1974):

$$
A I C_{i}=-2 \log L_{i}+2 p_{i}
$$

Where $\log L_{i}$ is the maximized log likelihood of the respective model $i$ at convergence and $p_{i}$ is the number of parameters obtained from each model. The model with the lowest AIC was taken into account as the most appropriate one. Total heritability, $h_{t}^{2}$ (Willham 1972) and maternal across year repeatability for ewe performance, $t_{m^{\prime}}$ (Gowane et al. 2010) were estimated as follows:

$$
\begin{aligned}
& h_{t}^{2}=\left(\sigma_{a}^{2}+0.5 \sigma^{2} m+1.5 \sigma_{a m}\right) / \sigma_{p}^{2} \\
& t_{m}=1 / 4 h_{d}^{2}+h_{m}^{2}+c^{2}+\left(m r_{a m} h\right)
\end{aligned}
$$

Components of the described formulas are explained in the footnote of Table 3. Genetic and phenotypic correlations were estimated using bivariate analyses applying the best model determined in univariate analyses. If the values of -2 log likelihood variance in the Simplex function were below $10^{-8}$, it was assumed that convergence had been achieved (Kushwaha et al. 2009).

\section{Results and discussion}

\section{Fixed effects}

Descriptive statistics of the studied traits are summarized in Table 1. Least squares means of the considered traits are shown in Table 2 . Single born, twin and triplet lambs constitute $52.68 \%, 38.14 \%$ and $9.18 \%$ of total lambs, respectively. As indicated in Table 2, birth year, sex and birth type of lambs had significant effects on all studied traits $(P<0.01)$. Dam age significantly influenced the considered pre-weaning traits $(P<0.01)$ but not post-weaning ones $(P>0.05)$. Weighing age at $3,6,9$ and 12 months of age as a linear covariate, had significant influence on $\mathrm{WW}, 6 \mathrm{MW}, 9 \mathrm{MW}$ and $\mathrm{YW}$, respectively.

Male lambs had higher pre-weaning growth rate and body weight than females at various ages. Such superiority can be partly ascribed to differences in the endocrine system of male and female lambs that tends to become more pronounced as lambs approach maturity. The significant effects of dam age on the studied traits can be explained to some extent by differences in maternal effects and maternal behaviour of ewes at different ages. Differences in 
Table 2

Least square means \pm standard error for the studied traits

\begin{tabular}{|c|c|c|c|c|c|c|c|}
\hline Sub-class & $\mathrm{BW}, \mathrm{kg}^{1}$ & WW, kg & ADG, g/day & KR & $6 \mathrm{MW}, \mathrm{kg}$ & $9 \mathrm{MW}, \mathrm{kg}$ & $\mathrm{YW}, \mathrm{kg}$ \\
\hline Sex & $* *$ & $* *$ & $* *$ & $* *$ & $* *$ & $* *$ & $* *$ \\
\hline Male & $5.13^{\mathrm{a}} \pm 0.06$ & $26.23^{\mathrm{a}} \pm 0.11$ & $220.57^{\mathrm{a}} \pm 2.41$ & $21.35^{a} \pm 0.12$ & $39.63^{\mathrm{a}} \pm 0.28$ & $44.35^{\mathrm{a}} \pm 0.35$ & $59.73^{a} \pm 0.72$ \\
\hline Female & $4.61^{b} \pm 0.04$ & $22.18^{\mathrm{a}} \pm 0.16$ & $191.07^{\mathrm{a}} \pm 2.29$ & $18.04^{a} \pm 0.16$ & $34.68^{b} \pm 0.22$ & $41.25^{b} \pm 0.29$ & $46.26^{b} \pm 0.26$ \\
\hline Birth type & $* *$ & $* *$ & $* *$ & $* *$ & $* *$ & $* *$ & $* *$ \\
\hline Single & $5.28^{\mathrm{a}} \pm 0.02$ & $29.52^{\mathrm{a}} \pm 0.15$ & $235.27^{\mathrm{a}} \pm 1.94$ & $21.18^{\mathrm{a}} \pm 0.13$ & $39.26^{\mathrm{a}} \pm 0.16$ & $45.38^{\mathrm{a}} \pm 0.28$ & $49.14^{\mathrm{a}} \pm 0.36$ \\
\hline Twin & $4.57^{b} \pm 0.05$ & $26.15^{b} \pm 13$ & $205.48^{b} \pm 1.04$ & $19.09^{b} \pm 0.10$ & $35.47^{\mathrm{b}} \pm 0.16$ & $41.59^{\mathrm{b}} \pm 0.38$ & $44.84^{b} \pm 0.58$ \\
\hline$>$ Triplet & $4.09^{\complement} \pm 0.02$ & $23.08^{c} \pm 0.26$ & $185.23^{c} \pm 2.87$ & $19.15^{b} \pm 0.17$ & $32.58^{b} \pm 0.59$ & $38.93^{b} \pm 0.17$ & $43.36^{b} \pm 0.94$ \\
\hline Dam age, year & $* *$ & $* *$ & $* *$ & $* *$ & ns & ns & ns \\
\hline 2 & $4.36^{c} \pm 0.01$ & $24.26 d \pm 0.16$ & $196.13^{b} \pm 2.85$ & $19.73^{c} \pm 0.25$ & $35.47^{\mathrm{a}} \pm 0.26$ & $42.25^{\mathrm{a}} \pm 0.37$ & $44.72^{\mathrm{a}} \pm 0.99$ \\
\hline 3 & $4.73 b^{c} \pm 0.02$ & $24.37 b^{c} \pm 0.26$ & $198.52^{b} \pm 2.71$ & $19.55^{b} \pm 0.26$ & $35.46^{\mathrm{a}} \pm 0.21$ & $43.16^{\mathrm{a}} \pm 0.83$ & $44.94^{a} \pm 0.72$ \\
\hline 4 & $4.83^{b} \pm 0.02$ & $24.38^{c} \pm 0.72$ & $193.10^{\mathrm{b}} \pm 3.86$ & $19.05^{\complement} \pm 0.28$ & $35.26^{\mathrm{a}} \pm 0.24$ & $44.27^{\mathrm{a}} \pm 0.36$ & $45.73^{a} \pm 0.72$ \\
\hline 5 & $4.93^{b} \pm 0.03$ & $26.17^{b} \pm 0.16$ & $210.82^{\mathrm{ab}} \pm 2.78$ & $20.07^{a} \pm 0.27$ & $36.71^{\mathrm{a}} \pm 0.53$ & $43.37^{\mathrm{a}} \pm 0.83$ & $45.93^{a} \pm 0.28$ \\
\hline 6 & $5.16^{\mathrm{a}} \pm 0.03$ & $28.94^{a} \pm 0.71$ & $225.61^{\mathrm{a}} \pm 3.83$ & $19.14^{c} \pm 0.36$ & $37.23^{\mathrm{a}} \pm 0.15$ & $46.17^{a} \pm 0.22$ & $46.93^{\mathrm{a}} \pm 0.82$ \\
\hline 7 & $5.10^{\mathrm{ab}} \pm 0.05$ & $27.26^{b} \pm 0.61$ & $206.51^{b} \pm 4.74$ & $19.21^{c} \pm 0.41$ & $38.27^{\mathrm{a}} \pm 0.15$ & $46.58^{\mathrm{a}} \pm 0.73$ & $46.82^{\mathrm{a}} \pm 0.12$ \\
\hline Birth year & $* *$ & $* *$ & $* *$ & $* *$ & $* *$ & $* *$ & $* *$ \\
\hline Birth date $^{2}$ & - & $0.21^{* *} \pm 0.01$ & - & - & $0.19^{* *} \pm 0.07$ & $0.16^{* *} \pm 0.04$ & $0.15^{* *} \pm 0.03$ \\
\hline
\end{tabular}

'Means with similar letters in each subclass within a column do not differ; ${ }^{*}$, ** significant effect at $P<0.05$ and $P<0.01$, respectively. ${ }^{2}$ regression coefficient on day of lambs' birth 
managing practices, feed availability, climatic conditions and breeding systems through years are possible reasons for significant effects of year on the considered traits. Competition for milk consumption among twins and triplets leads to a significant effect of birth type of lambs on the studied traits. Significant influences of investigated fixed effects on body weight of different sheep breed have been confirmed by others (Ghafouri-Kesbi et al. 2008, Abegaz et al. 2010).

\section{Model selection}

The AIC values for the studied traits under different tested models are presented in Table 3. The most appropriate model for BW and WW included direct additive genetic, maternal additive genetic and common litter effects, without considering covariance between direct additive and maternal additive genetic effects (Model 9). The most appropriate model for ADG and KR was similar to that of BW and WW, ignoring maternal additive genetic effects (Model 7). The model including direct additive genetic effects and maternal permanent environmental effects (Model 2) was determined as the best model for 6MW. Maternal effects had no influence on YW and resulted in selection of the simplest model, which included direct additive genetic effects as the sole random effects for 9MW and YW.

Table 3

AIC values for studied traits under different animal models with the best model in bold face

\begin{tabular}{lccccccc}
\hline Model & & \multicolumn{7}{c}{ Traits } \\
& BW & WW & ADG & KR & $6 M W$ & 9MW & YW \\
\hline Model 1 & 2151.346 & 450.425 & 2519.826 & 9445.481 & 845.215 & $\mathbf{7 3 1 6 . 3 2 6}$ & $\mathbf{1 2 4 2 1 2 . 1 6 7}$ \\
Model 2 & 2147.126 & 446.417 & 2513.439 & $\mathbf{9 4 3 5 . 4 5 7}$ & $\mathbf{8 4 4 . 2 1 4}$ & 7327.735 & 124214.673 \\
Model 3 & 2153.432 & 449.726 & 2516.370 & 9441.487 & 846.374 & 7328.156 & 124214.525 \\
Model 4 & 2155.387 & 451.167 & 2518.269 & 9443.047 & 847.254 & 7329.926 & 124216.156 \\
Model 5 & 2151.090 & 448.642 & 2515.256 & 9437.168 & 872.345 & 7424.725 & 124215.015 \\
Model 6 & 2150.211 & 450.625 & 2516.268 & 9439.356 & 871.732 & 7416.615 & 124242.156 \\
Model 7 & 2113.617 & 405.478 & $\mathbf{2 4 7 0 . 2 1 7}$ & 9443.099 & 873.356 & 7416.616 & 124243.235 \\
Model 8 & 2112.763 & 405.629 & 2472.374 & 9445.056 & 874.601 & 7417.672 & 124244.168 \\
Model 9 & $\mathbf{2 1 1 2 . 0 7 9}$ & $\mathbf{4 0 4 . 2 8 3}$ & 2471.302 & 9445.337 & 873.056 & 7418.175 & 124244.158 \\
Model 10 & 2114.427 & 486.160 & 2473.306 & 9447.728 & 870.581 & 7420.727 & 124245.145 \\
Model 11 & 2114.189 & 486.048 & 2473.302 & 9447.168 & 863.158 & 7416.549 & 124250.145 \\
Model 12 & 2116.165 & 488.300 & 2475.306 & 9449.269 & 864.917 & 7420.164 & 124252.156 \\
\hline
\end{tabular}

\section{Estimates of genetic parameters under univariate analysis}

Estimation of genetic parameters for the studied traits based on the best model under univariate analyses are presented in Table 4. Direct heritability values were estimated 0.13 , $0.19,0.18,0.05,0.16,0.18$ and 0.19 for BW, WW, ADG, KR, 6MW, 9MW and YW, respectively. Al-Shorepy (2001) pointed out that birth weight is an economically important trait because of its effect on pre-weaning growth rate and increase of economic success in any sheep production enterprise. 
Table 4

Genetic parameter estimates for traits studied fitting the most appropriate model

\begin{tabular}{lcccccccc}
\hline Traits & $\begin{array}{c}\text { Model } \\
\text { fitted }\end{array}$ & $h_{d}^{2} \pm \mathrm{SE}$ & $h_{m}^{2} \pm \mathrm{SE}$ & $c^{2} \pm \mathrm{SE}$ & $P^{2} \pm \mathrm{SE}$ & $h_{t}^{2}$ & $\mathrm{t}^{\mathrm{m}}$ & $\mathrm{S}_{p}^{2}$ \\
\hline BW & 9 & $0.13 \pm 0.03$ & $0.12 \pm 0.03$ & - & $0.05 \pm 0.02$ & 0.17 & 0.20 & 20.33 \\
WW & 9 & $0.19 \pm 0.02$ & $0.10 \pm 0.01$ & - & $0.12 \pm 0.04$ & 0.24 & 0.26 & 24.48 \\
ADG & 7 & $0.18 \pm 0.02$ & - & - & $0.14 \pm 0.03$ & 0.18 & 0.05 & 85.13 \\
KR & 2 & $0.05 \pm 0.01$ & - & $0.06 \pm 0.03$ & - & 0.05 & 0.07 & 10.51 \\
$6 \mathrm{MW}$ & 2 & $0.16 \pm 0.03$ & - & $0.06 \pm 0.02$ & - & 0.16 & 0.10 & 14.95 \\
9MW & 1 & $0.18 \pm 0.02$ & - & - & - & 0.18 & 0.04 & 14.53 \\
YW & 1 & $0.19 \pm 0.02$ & - & - & - & 0.19 & 0.05 & 30.40 \\
\hline
\end{tabular}

$\mathrm{s}_{p}^{2}$ : phenotypic variance; $h_{d}^{2}$ : direct heritability; $h_{m}^{2}$ : maternal heritability; $c^{2}$ : ratio of maternal permanent environmental effects to phenotypic variance; $R$ : ratio of common litter effects to phenotypic variance; SE: standard error; $h_{t}^{2}=$ Total heritability $=\left(\sigma^{2}{ }_{\mathrm{a}}+0.5 \sigma_{\mathrm{m}}^{2}+1.5 \sigma_{\mathrm{am}}\right) / \sigma_{\mathrm{p}}^{2} ; \mathrm{t}_{\mathrm{m}}=\left(1 / 4 h_{d}^{2}+h_{m}^{2}+c^{2}+\mathrm{m} \mathrm{r} \mathrm{rm}_{\mathrm{m}} \mathrm{h}\right)$

The low direct heritability estimated for BW denotes the fact that direct genetic effects constitute a negligible part of phenotypic variance for BW of Shal lambs, suggesting that slow genetic progress would be expected through direct selection. Such low direct heritability is possibly due to the inclusion of maternal effects in the selected model.

Maternal effects denote the mothering ability for milk production as well as intrauterine conditions and may be partitioned into genetic and non-genetic parts (Matika et al. 2003, Dobek et al. 2004). Maternal additive genetic effects disappeared after weaning. Maternal permanent environmental effects have influenced all pre-weaning traits except $K R$, whereas only $\mathrm{KR}$ and $6 \mathrm{MW}$ were affected by maternal temporary environmental ones.

Maternal heritability values were estimated as 0.12 and 0.10 for BW and WW, respectively. The estimates of maternal permanent environmental variance, as a proportion of phenotypic variance $\left(c^{2}\right)$ estimates, were low for $\mathrm{KR}(0.06)$ and $6 \mathrm{MW}(0.06)$. The estimate of common litter effect $\left(I^{2}\right)$ was 0.05 for BW, 0.12 for WW and 0.14 for ADG. The estimated value for maternal heritability of BW (0.13) was in concordance with estimates of Mohammadi et al. (2011) in Zandi sheep.

As it was expected, maternal effects formed an integral part of variation for BW, probably reflecting differences in the uterine conditions, mainly with respect to the quality and capacity of the uterine space for the growth of the foetus (Gowane et al. 2010). Maternal heritability estimate for BW was lower than direct heritability one. These results are in agreement with studies of several authors (Noor et al. 2001, Mohammadi et al. 2012). Maternal heritability estimate for WW was lower than the direct one $(0.10 \mathrm{vs}$. 0.19$)$ and generally agreed with the estimate of Zamani \& Mohammadi (2008) in Iranian Mehraban sheep (0.08). Lower estimates (Ozcan et al. 2005, Unal et al. 2006) also have been reported.

Estimated direct heritability values for WW (0.19) and ADG (0.18) were relatively similar in magnitude. Direct heritability estimate of WW accords well with literature (Gowane et al. 2010, Gowane et al. 2011, Mohammadi et al. 2012).

A low direct heritability value was estimated for KR (0.05), which generally accords well with those obtained by Matika et al. (2003) in Sabi sheep. The Kleiber ratio is proposed as an efficient selection criterion for feed efficiency under low-input range conditions and provides a good indication of how economically an animal grows (Mohammadi et al. 2011). Proportion 
of maternal temporary environmental variance, as a fraction of phenotypic variance $\left(\mathrm{I}^{2}\right)$ for BW, WW and ADG, was estimated as $0.05,0.12$ and 0.14 , respectively.

At post-weaning period direct heritability estimate decreased from 0.16 at six months of age to 0.18 at nine months of age and increased to a value of 0.19 at yearling age. Direct heritability estimated value for $6 \mathrm{MW}$ was generally concordant with estimates of Abegaz et al. (2005) in Horro sheep (0.18). Higher (Gowane et al. 2010) and lower (Eskandarinasab et al. 2010) values were also reported. Maternal permanent environmental effects may have arisen from uterine environmental and multiple birth effects on milk production of ewes, the level of nutrition at final stages of gestation and maternal behaviour of ewes.

There are little information regarding genetic parameters of 9MW in the literature and published values are mainly related to Iranian local sheep breeds (Mohammadi et al. 2011). Maternal effects disappeared after six months of age. The obtained direct heritability estimate of YW (0.19) generally agrees with the estimate of Mohammadi et al. (2012) in Makooei sheep (0.22).

Total heritability estimates $\left(h_{t}^{2}\right)$ were low to medium and ranging from 0.05 for KR to 0.24 for WW. Values of maternal across year repeatability for ewe performance $\left(t_{m}\right)$ generally decreased with age and varied from 0.04 for $9 \mathrm{MW}$ to 0.26 for WW. Total heritability estimates are model sensitive (Gowane et al. 2010). Abegaz et al. (2005) pointed out that total heritability is important for breeding when maternal effects are important for the expression of a trait, and is useful for the estimation of selection response based on phenotypic values. The obtained estimates of $h_{t}^{2}$ and $t_{m}$ for BW (0.17 and 0.20) and for WW (0.24 and 0.26) were in general agreement with estimated values reported by Gowane et al. (2010) in Malpura sheep. Such moderate estimates suggest scope of improvement in BW and WW through mass selection (Gowane et al. 2010). Estimates of $h_{t}^{2}$ for ADG and KR were 0.18 and 0.05 while those of $t_{m}$ were 0.05 and 0.07. Obtained estimates of $h_{t}^{2}$ for 6MW, 9MW and YW were $0.16,0.18$ and 0.19 whereas those of $t_{m}$ were $0.10,0.04$ and 0.05 for $6 \mathrm{MW}, 9 \mathrm{MW}$ and $\mathrm{YW}$, respectively. Estimates of $t_{m}$ for post-weaning body weights were generally higher than the estimated values by Gowane et al. (2010) in Malpura sheep.

\section{Correlation estimates}

Correlation estimates among the studied traits are presented in Table 5. There was no antagonistic relationship between the considered traits in terms of phenotypic, genetic and environmental correlations. Thus, selection for any of these traits will bring out a positive response to selection for others. Direct additive genetic correlations were positive and ranged from 0.09 for KR-YW to 0.80 for WW-ADG.

Birth weight had positive and low to medium direct genetic correlations with other studied traits, ranging from 0.17 (BW-KR) to 0.62 (BW-WW). Direct genetic correlation estimate of BW with other body weight traits decreased with age.

Direct genetic correlation estimate between WW and ADG was high and positive (0.80) and corresponds to estimates of Duguma et al. (2002) in Tygerhoek Merino sheep (0.99). Duguma et al. (2002) pointed out that WW and ADG are genetically the same trait. Thus, selection can be carried out based on either one of them. Medium and positive direct genetic 
Table 5

Correlation estimates among body weight traits

\begin{tabular}{lcccccc}
\hline Traits & $r_{p}$ & $r_{a}$ & $r_{m}$ & $r_{c}$ & $r_{1}$ & $r_{e}$ \\
\hline BW-WW & $0.29 \pm 0.05$ & $0.62 \pm 0.12$ & $0.30 \pm 0.09$ & - & $0.79 \pm 0.22$ & $0.18 \pm 0.07$ \\
BW-ADG & $0.28 \pm 0.10$ & $0.22 \pm 0.16$ & - & - & $0.68 \pm 0.18$ & $0.17 \pm 0.09$ \\
BW-KR & $0.24 \pm 0.14$ & $0.17 \pm 0.04$ & - & - & - & $0.11 \pm 0.08$ \\
BW-6MW & $0.28 \pm 0.04$ & $0.53 \pm 0.12$ & - & - & - & $0.23 \pm 0.10$ \\
BW-9MW & $0.23 \pm 0.02$ & $0.42 \pm 0.11$ & - & - & - & $0.16 \pm 0.08$ \\
BW-YW & $0.20 \pm 0.09$ & $0.32 \pm 0.15$ & - & - & - & $0.19 \pm 0.07$ \\
WW-ADG & $0.87 \pm 0.15$ & $0.80 \pm 0.15$ & - & - & - & $0.69 \pm 0.18$ \\
WW-KR & $0.62 \pm 0.14$ & $0.64 \pm 0.12$ & - & - & - & $0.58 \pm 0.12$ \\
WW-6MW & $0.39 \pm 0.05$ & $0.52 \pm 0.11$ & - & - & - & $0.43 \pm 0.10$ \\
WW-9MW & $0.28 \pm 0.14$ & $0.39 \pm 0.15$ & - & - & - & $0.25 \pm 0.14$ \\
WW-YW & $0.23 \pm 0.11$ & $0.33 \pm 0.09$ & - & - & - & $0.20 \pm 0.13$ \\
ADG-KR & $0.43 \pm 0.16$ & $0.79 \pm 0.19$ & - & - & - & $0.37 \pm 0.16$ \\
ADG-6MW & $0.34 \pm 0.11$ & $0.46 \pm 0.16$ & - & - & - & $0.45 \pm 0.19$ \\
ADG-9MW & $0.28 \pm 0.13$ & $0.39 \pm 0.15$ & - & - & - & $0.36 \pm 0.14$ \\
ADG-YW & $0.19 \pm 0.09$ & $0.29 \pm 0.15$ & - & - & - & $0.28 \pm 0.14$ \\
KR-6MW & $0.42 \pm 0.12$ & $0.30 \pm 0.16$ & - & $0.27 \pm 0.12$ & - & $0.29 \pm 0.15$ \\
KR-9MW & $0.21 \pm 0.10$ & $0.14 \pm 0.13$ & - & - & - & $0.35 \pm 0.13$ \\
KR-YW & $0.18 \pm 0.05$ & $0.09 \pm 0.08$ & - & - & - & $0.06 \pm 0.03$ \\
6MW-9MW & $0.52 \pm 0.13$ & $0.62 \pm 0.18$ & - & - & - & $0.37 \pm 0.11$ \\
6MW-YW & $0.43 \pm 0.06$ & $0.45 \pm 0.17$ & - & - & - & $0.29 \pm 0.10$ \\
9MW-YW & $0.36 \pm 0.12$ & $0.45 \pm 0.13$ & - & - & - & $0.39 \pm 0.16$ \\
\hline
\end{tabular}

$r_{p}$ : phenotypic correlation; $r_{a}$ : direct genetic correlation; $r_{m}$ : maternal genetic correlation; $r_{c}$ : maternal permanent environmental correlation; $r_{i}$ : common litter effect correlation; $r_{\mathrm{e}}$ : environmental correlation

correlations were found between WW and other traits (Table 4). Estimated values for direct genetic correlations of WW with 6MW, 9MW and YW generally agreed with estimates of Gowane et al. (2010) in Malpura sheep. Weaning weight had a medium and positive direct genetic correlation with KR (0.64).

A relatively high direct genetic correlation estimate was obtained for ADG-KR (0.79) which was consistent with those obtained by Mohammadi et al. (2012) in Zandi sheep. Direct genetic correlations of post-weaning body weight traits with ADG were higher than those of post-weaning ones with KR. Direct genetic correlation estimate of 6MW-YW (0.45) was similar to the estimate of Miraei-Ashtiani et al. (2007) in Sangsari sheep (0.55). The existence of positive direct genetic correlations among the studied traits suggests that genetic factors which influence these traits were in similar direction.

An estimate of 0.30 for maternal genetic correlation between BW and WW was found, indicating that maternal additive genetic effects, which favour the growth of foetus, could have some favourable effects on post-natal growth traits of Shal lambs. Body weight from birth to weaning is influenced by similar genes of the ewe in terms of maternal genetic effects. 
Positive and medium estimated value for maternal permanent environmental correlation between KR and 6MW (0.27) suggest that the existence of good management conditions and favourable maternal behaviour results in a beneficial effect on body weight of lambs at 6 months of age (Gowane et al. 2010). Maternal temporary environmental correlations were high in magnitude and found only among pre-weaning traits except KR.

Estimates of phenotypic correlation were positive and ranged from low ( 0.18 for KR-YW) to high ( 0.87 for WW-ADG) values. Environmental correlation estimates were ranged from 0.06 for KR-YW to 0.69 for WW-ADG. Corresponding correlation estimates generally agreed with those of Abegaz et al. (2005) in Horro sheep. Positive genetic phenotypic and environmental correlations among body weight traits indicate that there is no genetic, phenotypic and environmental antagonistic relationship between the considered traits.

In conclusion, the present study contributes to the comparison of different models for estimation of (co)variance components and genetic parameters in Shal sheep. There was found low direct genetic variation for all studied traits. Thus, a relatively low genetic gain would be expected through mass selection and the investigation of indirect selection criteria such as reproduction traits for improvement of growth traits is of paramount importance. Only BW and WW were affected by maternal additive genetic effects. It was obviously shown that the maternal environmental effects should be portioned into permanent and temporary components until six months of age.

\section{References}

Abegaz S, van Wyk JB, Olivier JJ (2005) Model comparisons and genetic and environmental parameter estimates of growth and the Kleiber ratio in Horro sheep. S Afr J Anim Sci 35, 30-40

Abegaz S, van Wyk JB, Olivier JJ (2010) Estimates of (co)variance function for growth to yearling in Horro sheep of Ethiopia using random regression model. Arch Tierz 53, 689-700

Akaike H (1974) A new look at the statistical model identification. IEEE Trans Automat Contr 19, 716-723

Al-Shorepy SA (2001) Estimates of genetic parameters for direct and maternal effects on birth weight of local sheep in United Arab Emirates. Small Rumin Res 39, 219-224

Di J, Zhang Y, T KC, Lazate, Liu JF, Xu XM, Zhang YJ, Zhang TH (2011) Estimation of (co)variance components and genetic parameters for growth and wool traits of Chinese superfine merino sheep with the use of a multi-trait animal model. Livest Sci 138, 278-288

Dobek A, Wojtowski J, Szwaczkowski T, Molinski K, Gut A (2004) Genetic variability for birth and fourth week weights in sheep. Arch Tierz 47 (Special Issue), 64-72

Duguma G, Schoeman SJ, Cloete SWP, Jordaan GF (2002) Genetic parameter estimates of early growth traits in the Tygerhoek Merino flock. S Afr J Anim Sci 32, 66-75

Eskandarinasab M, Ghafouri-Kesbi F, Abbasi MA (2010) Different models for evaluation of growth traits and Kleiber ratio in an experimental flock of Iranian fat-tailed Afshari sheep. J Anim Breed Genet 127, 26-33

Freking BA, Leymaster KA (2004) Evaluation of Dorset, Finnsheep, Romanov, Texel, and Montadale breeds of sheep: IV. Survival, growth, and carcass traits of F1 lambs. J Anim Sci 82, 144-153

Ghafouri-Kesbi F, Eskandarinasab M, Hassanabadi A (2008) Estimation of genetic parameters for lamb weight at various ages in Mehraban sheep. Ital J Anim Sci 7, 95-103

Gowane GR, Chopra A, Prakash V, Arora AL (2010) Estimates of (co)variance components and genetic parameters for body weights and first greasy fleece weight in Malpura sheep. Livest Sci 131, 94-101

Gowane GR, Chopra A, Prince LLL, Mishra AK, Arora AL (2011) Genetic analysis for growth traits of prolific GarolexMalpura (GM) sheep. Trop Anim Health Prod 43, 299-303 
Gilmour AR, Bullis BR, Welham SJ, Thompson R (2000) ASREML Reference Manual. NSW Agric Biometric Bulletin 3, Orange Agriculture Institute, Orange, NSW, Australia

Kushwaha BP, Mandal A, Arora AL, Kumar R, Kumar S, Notter DR (2009) Direct and maternal (co)variance components and heritability estimates for body weights in Chokla sheep. J Anim Breed Genet 126, 278-287

Matika O, van Wyk JB, Erasmus GJ, Baker RL (2003) Genetic parameter estimates in Sabi sheep. Livest Prod Sci 79, $17-28$

Miraei-Ashtiani SR, Seyedalian SAR, Moradi Shahrbabak M (2007) Variance components and heritabilities for body weight traits in Sangsari sheep, using univariate and multivariate animal models. Small Rumin Res 73, 109-114

Mohammadi K, Rashidi A, Mokhtari MS, Beigi Nassiri MT (2011) The estimation of (co)variance components for growth traits and Kleiber ratios in Zandi sheep. Small Rumin Res 99, 116-121

Mohammadi H, Moradi Shahrebabak M, Vatankhah M, Moradi Shahrebabak H (2102) Direct and maternal (co) variance components, genetic parameters, and annual trends for growth traits of Makooei sheep in Iran. Trop Anim Health Prod 45, 185-191

Noor RR, Djajanegara A, Schüler L (2001) Selection to improve birth and weaning weight of Javanese Fat Tailed Sheep. Arch Tierz 44, 649-659

Ozcan M, Ekiz B, Yilmaz A, Ceyhan A (2005) Genetic parameter estimates for lamb growth traits and greasy fleece weight at first shearing in Turkish Merino sheep. Small Rumin Res 56, 215-222

Prince LLL, Gowane GR, Chopra A, Arora AL (2010) Estimates of (co)variance components and genetic parameters for growth traits of Avikalin sheep. Trop Anim Health Prod 42, 1093-1101

Tavakolian J (1999) [The genetic resources of native farm animals of Iran.] Animal Science Research Institute of Iran [in Persian]

Tosh R, Kemp A (1994) Estimation of variance components for lamb weights in three sheep populations. J Anim Sci 72, 1184-1190

Unal N, Akcapinar H, Atasoy F, Aytac M (2006) Some reproductive and growth traits of crossbred genotypes produced by crossing local sheep breeds of Kivircik $x$ White Karaman and Chios $x$ White Karaman in steppe condition. Arch Tierz 49, 55-63

Van Wyk JB, Fair MD, Cloete SWP (2003) Revised models and genetic parameter estimates for production and reproduction traits in the Elsenburg Dormer sheep stud. S Afr J Anim Sci 33, 213-222

Vatankhah M, Moradi-Shahrbabak M, Nejati-Javaremi A, Miraei-Ashtiani S R, Vaez-Torshizi R (2004) [A review of sheep breeding in Iran]. In: Proceedings of the First Congress on Animal and Aquatic Sciences. Vol. 2, Tehran, Iran, 590-596 [in Persian]

Willham RL (1972) The Role of Maternal Effects in Animal Breeding: III. Biometrical Aspects of Maternal Effects in Animals. J Anim Sci 35, 1288-1293

Zamani P, Mohammadi H (2008) Comparison of different models for estimation of genetic parameters of early growth traits in the Mehraban sheep. J Anim Breed Genet 125, 29-34 